\title{
ON THE ZEROS OF STIELTJES AND VAN VLECK POLYNOMIALS
}

\author{
BY
}

NEYAMAT ZAHEER AND MAHFOOZ ALAM $\left({ }^{1}\right)$

Dedicated to the University of Wisconsin-Milwaukee Distinguished Professor

MORRIS MARDEN ON THE OCCASION OF HIS RETIREMENT ON MAY 9, 1975

\begin{abstract}
Stieltjes and Van Vleck polynomials arise in the study of the polynomial solutions of the generalized Lamé differential equation. Our object is to generalize a theorem due to Marden on the location of the zeros of Stieltjes and Van Vleck polynomials. In fact, our generalization is two-fold: Firstly, we employ sets which are more general than the ones used by Marden for prescribing the location of the complex constants occurring in the Lamé differential equation; secondly, Marden deals only with the standard form of the said differential equation, whereas our result is equally valid for yet another form of the same differential equation. The part of our main theorem concerning Stieltjes polynomials may also be regarded as a generalization of Lucas' theorem to systems of partial fraction sums.
\end{abstract}

1. Introduction. A generalized Lamé differential equation is a second order linear differential equation of the form

$$
\frac{d^{2} w}{d z^{2}}+\left[\frac{\sum_{j=1}^{p} \alpha_{j}}{\left(z-a_{j}\right)}\right] \cdot \frac{d w}{d z}+\frac{\Phi(z)}{\Pi_{j=1}^{p}\left(z-a_{j}\right)} \cdot w=0,
$$

where $\Phi(z)$ is a polynomial of degree at most $(p-2)$ and $\alpha_{j}, a_{j}$ are complex constants. Heine [3] showed that there exist at most $C(n+p-2, p-2)$ polynomials $V(z)$ such that for $\Phi(z)=V(z)$ equation (1.1) has a polynomial solution $S(z)$ of degree $n$. Each polynomial $V(z)$ and the corresponding polynomial $S(z)$, associated with the differential equation (1.1), are, respectively, called [1, p. 37] a Van Vleck polynomial and a Stieltjes polynomial.

We shall be primarily interested in determining the location of the zeros of the systems of polynomials that arise in the study of the polynomial solutions of the differential equation

Received by the editors March 17, 1975 and, in revised form, December 8, 1975.

AMS (MOS) subject classifications (1970). Primary 30A08; Secondary 33A70.

Key words and phrases. Generalized Lamé differential equations, Stieltjes polynomials, Van Vleck polynomials.

(') Work of the second author was supported by the University Grants Commission (Government of India) Research Fellowship. 


$$
\begin{aligned}
& \frac{d^{2} w}{d z^{2}}+\left[\sum_{j=1}^{p} \alpha_{j}\right.\left.\left\{\frac{\Pi_{i=1}^{n_{j}-1}\left(z-b_{j t}\right)}{\prod_{s=1}^{n_{j}}\left(z-a_{j s}\right)}\right\}\right] \\
& \cdot \frac{d w}{d z}+\left[\frac{\Phi(z)}{\Pi_{j=1}^{p} \Pi_{s=1}^{n_{j}}\left(z-a_{j s}\right)}\right] \cdot w=0,
\end{aligned}
$$

where $\Phi(z)$ is a polynomial of degree at most $\left(n_{1}+n_{2}+\cdots+n_{p}-2\right)$ and $a_{j s}, b_{j t}$ and $\alpha_{j}$ are suitable complex constants. We may, however, note that the differential equation (1.2) can always be written in the form (1.1) by expressing each fraction (in the coefficient of $d w / d z$ ) into its partial fractions, and that (1.2) is indeed of the form (1.1) in case $n_{j}=1$ for all values of $j$. Consequently, there do exist Stieltjes polynomials $S(z)$ and Van Vleck polynomials $V(z)$ associated with the differential equation (1.2).

In order to avoid repetitions of long expressions and unnecessary details in the treatment of our proofs throughout the next section, we shall freely use the following abbreviations and notations: We shall denote by $S(z)$ the $n$ th-degree Stieltjes polynomial corresponding to a Van Vleck polynomial $V(z)$ associated with the differential equation (1.2) or (1.1); the zeros of $V(z)$, $S(z)$, and $S^{\prime}(z)$ will be denoted by $t_{j}, z_{j}$, and $z_{j}^{\prime}$, respectively. We shall write

$$
f_{j}(z)=\prod_{t=1}^{n_{j}-1}\left(z-b_{j t}\right), \quad g_{j}(z)=\prod_{s=1}^{n_{j}}\left(z-a_{j s}\right), \quad h_{j}(z)=\frac{f_{j}(z)}{g_{j}(z)}
$$

for every $j=1,2, \ldots, p$ (with the convention that $f_{j}(z) \equiv 1$ for $n_{j}=1$ ), and

$$
F(z)=\sum_{j=1}^{p} \alpha_{j} \cdot h_{j}(z)
$$

2. A generalization of Marden's theorem. In this section we generalize a theorem due to Marden $([1$, Theorem $(9,1)]$ or $[2])$ on the location of the zeros of Stieltjes polynomials and of Van Vleck polynomials associated with the differential equation (1.1). Our generalization is two-fold: Firstly, we use sets which are more general than the ones used by Marden for prescribing the location of the points $a_{j}$ (cf. (1.1)); secondly, Marden's theorem deals with the polynomials $S(z)$ and $V(z)$ in relation to (1.1) only, whereas our result is equally valid for both forms (1.1) and (1.2).

In order to prove our main theorem, we need to establish the following lemmas on the zeros of the polynomials $S(z)$ and $V(z)$.

LEMMA (2.1). Every zero $z_{k}$ of an nth-degree Stieltjes polynomial $S(z)$, associated with the differential equation (1.2), is either one of the points $a_{j s}$ $\left(1 \leqslant j \leqslant p, 1 \leqslant s \leqslant n_{j}\right)$ or satisfies the equation 


$$
\frac{1}{2} F\left(z_{k}\right)+\sum_{j \neq k ; j=1}^{n} \frac{1}{z_{k}-z_{j}}=0
$$

where $F(z)$ is as defined by (1.4).

Proof. Let $S(z)=\prod_{j-1}^{n}\left(z-z_{j}\right)$ be a Stieltjes polynomial corresponding to a Van Vleck polynomial $V(z)$. Then (cf. abbreviations (1.3)-(1.4)) the differential equation (1.2) can be written as

$$
S^{\prime \prime}(z)+F(z) \cdot S^{\prime}(z)+\left[\frac{V(z)}{\prod_{j=1}^{p} g_{j}(z)}\right] \cdot S(z)=0 .
$$

If $z_{k} \neq a_{j s}\left(1<j<p, 1<s<n_{j}\right)$ is a zero of $S(z)$, then equation (2.2) becomes

$$
S^{\prime \prime}\left(z_{k}\right)+F\left(z_{k}\right) \cdot S_{.}^{\prime}\left(z_{k}\right)=0 .
$$

If $z_{k} \neq a_{j s}$, then two cases are to be considered:

Case 1. $S^{\prime}\left(z_{k}\right)=0$. Using equation (2.3) and successively differentiating equation (2.2) we observe that $S^{(m)}\left(z_{k}\right)=0$ for all $m$, which contradicts the fact that the degree of $S(z)$ is $n$. Hence, this case is impossible to happen.

Case 2. $S^{\prime}\left(z_{k}\right) \neq 0$. Since $z_{k}$ is a nonrepeated zero of $S(z)$ in this case, we can write $S(z)=\left(z-z_{k}\right) T(z)$, where $T\left(z_{k}\right) \neq 0$. Therefore $S^{\prime}\left(z_{k}\right)=T\left(z_{k}\right)$ and $S^{\prime \prime}\left(z_{k}\right)=2 T^{\prime}\left(z_{k}\right)$. Consequently, due to (2.3), we have

$$
-F\left(z_{k}\right)=\frac{S^{\prime \prime}\left(z_{k}\right)}{S^{\prime}\left(z_{k}\right)}=2 \frac{T^{\prime}\left(z_{k}\right)}{T\left(z_{k}\right)}=2 \cdot \sum_{j \neq k, j=1}^{n} \frac{1}{z_{k}-z_{j}} .
$$

That is,

$$
\frac{1}{2} \cdot F\left(z_{k}\right)+\sum_{j \neq k ; j=1}^{n} \frac{1}{z_{k}-z_{j}}=0 .
$$

This establishes our claim regarding the zeros of $S(z)$.

LEMMA (2.2). Every zero $t_{k}$ of $a$ Van Vleck polynomial V(z), associated with the differential equation (1.2), if not an $a_{j s}$ is either one of the zeros of $S^{\prime}(z)$ or satisfies the equation

$$
F\left(t_{k}\right)+\sum_{j=1}^{n-1} \frac{1}{t_{k}-z_{j}^{\prime}}=0,
$$

$z_{j}^{\prime}$ being the zeros of $S^{\prime}(z)$.

Proof. If $t_{k}$ is a zero of $V(z)$ and if $S(z)$ is a corresponding Stieltjes polynomial, then from (1.2) we get

$$
S^{\prime \prime}\left(t_{k}\right)+F\left(t_{k}\right) \cdot S^{\prime}\left(t_{k}\right)=0
$$

If $t_{k} \neq a_{j s}$ and $S^{\prime}\left(t_{k}\right) \neq 0$, then 


$$
\frac{S^{\prime \prime}\left(t_{k}\right)}{S^{\prime}\left(t_{k}\right)}=\sum_{j=1}^{n-1} \frac{1}{\left(t_{k}-z_{j}^{\prime}\right)},
$$

where $z_{j}^{\prime}(1 \leqslant j \leqslant n-1)$ are the zeros of $S^{\prime}(z)$. Thus, from (2.5) it follows that

$$
-F\left(t_{k}\right)=\sum_{j=1}^{n-1} \frac{1}{t_{k}-z_{j}^{\prime}} .
$$

That is, $t_{k}$ satisfies equation (2.4). The proof is now complete.

We now proceed to prove our main theorem.

THEOREM (2.3). Let $E$ be an ellipse with centre at the point $c$, semimajor axis as $a$, and eccentricity $e$. Let $0 \leqslant \gamma<\pi / 2$ and

$$
\lambda=\max \left\{1,\left(1-e^{2} / 2\right)^{1 / 2} \sec \gamma\right\} .
$$

If $\left|\arg \alpha_{j}\right| \leqslant \cos ^{-1}(1 / \lambda)$ for every $j=1,2, \ldots, p$, and if all the points $a_{j s}, b_{j t}$ (occurring in equation (1.2)) lie on or inside $E$, then the zeros of each Stieltjes polynomial $S(z)$ and the zeros of each Van Vleck polynomial $V(z)$, associated with the differential equation (1.2), lie in the circular region

$$
|z-c| \leqslant a \sec \left[\left\{(q-1) \pi+\cos ^{-1}(1 / \lambda)\right\} /(2 q-1)\right],
$$

where $q=\max \left\{n_{1}, n_{2}, \ldots, n_{p}\right\}$.

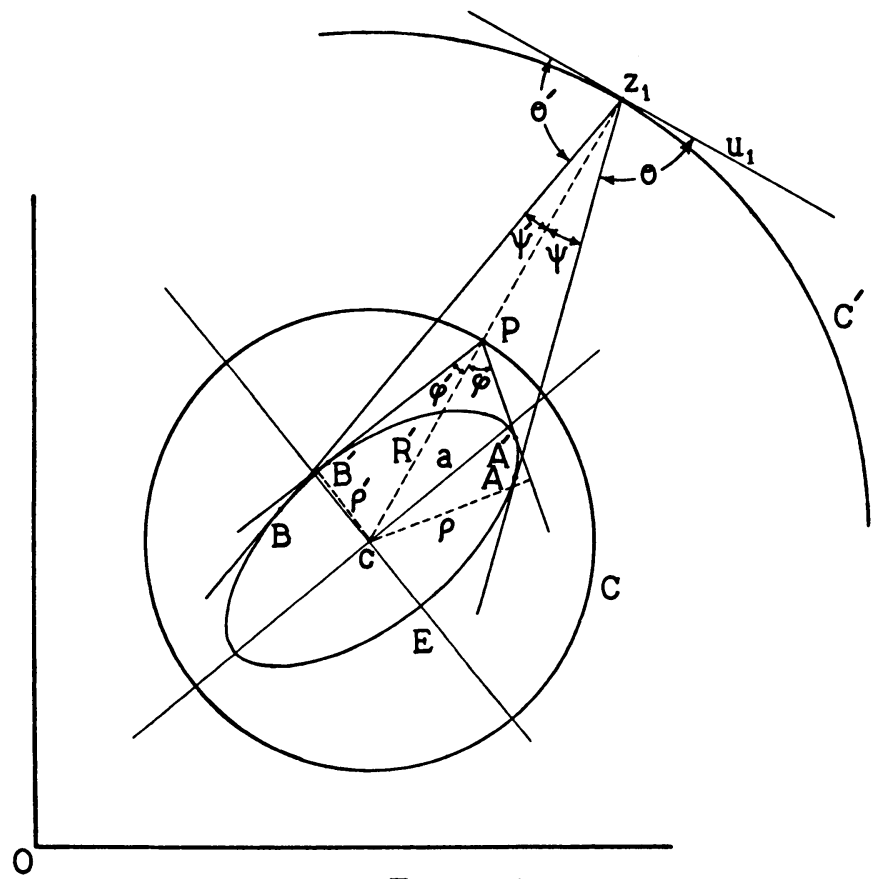

FIGURE 1 
Proof. Let us draw a circle $C$ with centre at the point $c$ and radius as

$$
R^{\prime}=a \sec \left[\left\{(q-1) \pi+\cos ^{-1}(1 / \lambda)\right\} /(2 q-1)\right] .
$$

Suppose, on the contrary, that one or more zeros $z_{k}$ of $S(z)$ lie outside the circle $C$ and that $z_{1}$ is the one farthest from the centre $c$, so that all the zeros of $S(z)$ lie on or inside the circle $C^{\prime}$ given by $|z-c|=\left|z_{1}\right|$. Let $\theta, \theta^{\prime}$, respectively, denote the acute angles which the tangents from $z_{1}$ to the ellipse $E$ make with the tangent at the point $z_{1}$ on $C^{\prime}$ (see Figure 1). Now join $z_{1}$ to the centre $c$ of the ellipse by a straight line, cutting the circle $C$ at a point $P$ (say). Now draw the tangents $P A^{\prime}$ and $P B^{\prime}$ from the point $P$ to the ellipse $E$. Let $\psi, \psi^{\prime}$ (resp. $\varphi, \varphi^{\prime}$ ) denote, respectively, the angles which the tangents from the point $z_{1}$ (resp. $P$ ) to the ellipse make with the line joining the points $z_{1}$ and $c$ (Figure 1). Since $z_{1}$ is outside $C$, we see that $\psi<\varphi$ and $\psi^{\prime}<\varphi^{\prime}$. Let $\varphi_{0}$ (resp. $\varphi_{0}^{\prime}$ ) be the maximum value of $\varphi$ (resp. $\varphi^{\prime}$ ) corresponding to all possible positions that $z_{1}$ could take on the circle $C^{\prime}$. If $\rho$ (resp. $\rho^{\prime}$ ) denotes the length of the perpendicular dropped from the centre $c$ upon the tangent $P A^{\prime}$ (resp. $P B^{\prime}$ ), we see that $\sin \varphi=\rho / R^{\prime}$ (resp. $\sin \varphi^{\prime}=\rho^{\prime} / R^{\prime}$ ) and that $\varphi$ (resp. $\varphi^{\prime}$ ) takes a maximum value when $\rho=a\left(\right.$ resp. $\left.\rho^{\prime}=a\right)$. Therefore,

$$
\sin \varphi_{0}=\sin \varphi_{0}^{\prime}=\cos \left\{(q-1) \pi+\cos ^{-1}(1 / \lambda)\right\} /(2 q-1) .
$$

Now, the definition of $\lambda$ implies that $0 \leqslant \cos ^{-1}(1 / \lambda) \leqslant \gamma<\pi / 2$. Using these inequalities, we can easily verify that

$$
0 \leqslant\left\{(q-1) \pi+\cos ^{-1}(1 / \lambda)\right\} /(2 q-1)<\pi / 2
$$

and that

$$
\frac{(q-1) \pi+\cos ^{-1}(1 / \lambda)}{(2 q-1)} \geqslant \frac{\left(n_{j}-1\right) \pi+\cos ^{-1}(1 / \lambda)}{\left(2 n_{j}-1\right)}, \quad 1 \leqslant j \leqslant p .
$$

Since, $\psi<\varphi \leqslant \varphi_{0}$ and $\psi^{\prime}<\varphi^{\prime} \leqslant \varphi_{0}^{\prime}$, from (2.6) and (2.7) we conclude that

$$
\theta=(\pi / 2-\psi)>\left(\pi / 2-\varphi_{0}\right)=\left\{(q-1) \pi+\cos ^{-1}(1 / \lambda)\right\} /(2 q-1)
$$

and

$$
\theta^{\prime}=\left(\pi / 2-\psi^{\prime}\right)>\left(\pi / 2-\varphi_{0}^{\prime}\right)=\left\{(q-1) \pi+\cos ^{-1}(1 / \lambda)\right\} /(2 q-1) .
$$

Consequently, due to inequalities (2.8), we have

$$
\theta, \theta^{\prime}>\frac{\left(n_{j}-1\right) \pi+\cos ^{-1}(1 / \lambda)}{\left(2 n_{j}-1\right)}=\mu_{j} \quad \text { (say) } \forall j=1,2, \ldots, p .
$$

Let $S(z)=\prod_{j=1}^{n}\left(z-z_{j}\right)$. If any zero $z_{k}$ of $S(z)$ is one of the points $a_{j s}$, then it lies in $C$ and we are done. However, if a zero $z_{k}$ is none of the points $a_{j s}$, then (cf. Lemma (2.1)) $z_{k}$ satisfies equation (2.1). In particular (for $k=1$ ), we have 


$$
\frac{1}{2} \cdot F\left(z_{1}\right)+\sum_{j=2}^{n} \frac{1}{z_{1}-z_{j}}=0
$$

Therefore,

$$
\frac{1}{2} \cdot F\left(z_{1}\right) \cdot\left(z_{1}-u_{1}\right)+\sum_{j=2}^{n}\left(\frac{z_{1}-u_{1}}{z_{1}-z_{j}}\right)=0,
$$

where $u_{1}$ is any point on the tangent drawn to the circle $|z-c|=\left|z_{1}\right|$ at the point $z_{1}$, i.e.,

$$
\sum_{j=1}^{p}\left[\frac{\alpha_{j}}{2} \cdot \prod_{t=1}^{n_{j}-1}\left(\frac{z_{1}-b_{j t}}{z_{1}-u_{1}}\right) \cdot \prod_{s=1}^{n_{j}}\left(\frac{z_{1}-u_{1}}{z_{1}-a_{j s}}\right)\right]+\sum_{j=2}^{n}\left(\frac{z_{1}-u_{1}}{z_{1}-z_{j}}\right)=0
$$

Since all the zeros $z_{k}$ lie in $|z-c| \leqslant\left|z_{1}\right|$, we get

$$
0<\arg \left(\frac{z_{1}-u_{1}}{z_{1}-z_{j}}\right)<\pi \quad \forall j=2,3, \ldots, n .
$$

Next, we also observe that the inequalities

$$
-\left(\pi-\theta^{\prime}\right)<\arg \left(\frac{z_{1}-b_{j t}}{z_{1}-u_{1}}\right)<-\theta
$$

and

$$
\theta<\arg \left(\frac{z_{1}-u_{1}}{z_{1}-a_{j s}}\right)<\pi-\theta^{\prime}
$$

hold for every $j=1,2, \ldots, p ; s=1,2, \ldots, n_{j} ; t=1,2, \ldots, n_{j-1}$. Inequalities (2.9), (2.12), and (2.13) imply that

$$
-\left(n_{j}-1\right)\left(\pi-\mu_{j}\right)<\arg \left[\prod_{t=1}^{n_{j}-1}\left(\frac{z_{1}-b_{j t}}{z_{1}-u_{1}}\right)\right]<-\left(n_{j}-1\right) \mu_{j}
$$

and

$$
n_{j} \mu_{j}<\arg \left[\prod_{s=1}^{n_{j}}\left(\frac{z_{1}-u_{1}}{z_{1}-a_{j s}}\right)\right]<n_{j}\left(\pi-\mu_{j}\right)
$$

for all values of $j=1,2, \ldots, p$. Using the above inequalities and the value of $\mu_{j}$ from (2.9), a simple calculation yields the following inequalities:

$$
\begin{aligned}
\cos ^{-1}(1 / \lambda) & <\arg \left[\prod_{t=1}^{n_{j}-1}\left(\frac{z_{1}-b_{j t}}{z_{1}-u_{1}}\right) \cdot \prod_{s=1}^{n_{j}}\left(\frac{z_{1}-u_{1}}{z_{1}-a_{j s}}\right)\right] \\
& <\pi-\cos ^{-1}(1 / \lambda)
\end{aligned}
$$


for every $j=1,2, \ldots, p$. In view of (2.14) and the hypothesis on $\alpha_{j}$, we conclude that

$$
0<\arg \left[\frac{\alpha_{j}}{2} \cdot \prod_{t=1}^{n_{j}-1}\left(\frac{z_{1}-b_{j t}}{z_{1}-u_{1}}\right) \cdot \prod_{s=1}^{n_{j}}\left(\frac{z_{1}-u_{1}}{z_{1}-a_{j s}}\right)\right]<\pi
$$

for every $j=1,2, \ldots, p$. Hence, in the light of inequalities (2.11) and (2.15), the imaginary part of each term on the left-hand side of equation (2.10) is positive. This contradicts the fact that $z_{1}$ satisfies equation (2.10). Therefore, all the zeros of $S(z)$ lie on or inside the circle $C$.

Now, we prove the second part of our theorem for a Van Vleck polynomial $V(z)$ corresponding to an $n$ th-degree Stieltjes polynomial $S(z)$. Proceeding as in the case of Stieltjes polynomials and assuming $t_{1}$ to be a zero of $V(z)$ lying outside the circle $C$ and farthest from the centre $c$, we see that all the zeros of $V(z)$ lie on or inside the circle $C^{\prime}$ given by $|z-c|=\left|t_{1}\right|$. Our previous diagram (Figure 1) remains the same except that $t_{1}$ replaces $z_{1}$. If $t_{1}=a_{j s}$, then $t_{1}$ is in $E$ and, hence, in $C$. In case $t_{1}$ is a zero of $S^{\prime}(z)$, then the Lucas theorem [1, Theorem $(6,2)],[4],[5],[6]$, together with the first part of our theorem (just proved), implies that $t_{1}$ lies in $C$ and, hence, the theorem follows. In case $t_{1}$ is not a zero of $S^{\prime}(z)$, then (cf. Lemma (2.2)) it satisfies equation (2.4) with $k=1$. Hence

$$
F\left(t_{1}\right)+\sum_{j=1}^{n-1} \frac{1}{t_{1}-z_{j}^{\prime}}=0
$$

Therefore,

$$
F\left(t_{1}\right) \cdot\left(t_{1}-u_{1}\right)+\sum_{j=1}^{n-1} \frac{t_{1}-u_{1}}{t_{1}-z_{j}^{\prime}}=0,
$$

where $u_{1}$ is any point on the tangent to the circle $|z-c|=\left|t_{1}\right|$ at the point $t_{1}$, i.e.,

$$
\sum_{j=1}^{p}\left(\alpha_{j}\right)\left\{\prod_{t=1}^{n_{j}-1}\left(\frac{t_{1}-b_{j t}}{t_{1}-u_{1}}\right) \cdot \prod_{s=1}^{n_{j}}\left(\frac{t_{1}-u_{1}}{t_{1}-a_{j s}}\right)\right\}+\sum_{j=1}^{n-1} \frac{t_{1}-u_{1}}{t_{1}-z_{j}^{\prime}}=0
$$

Since the points $z_{j}^{\prime}$ lie in $C$, we may replace $z_{j}$ by $z_{j}^{\prime}$ and $z_{1}$ by $t_{1}$ in inequalities (2.11) and (2.15) and obtain

$$
0<\arg \left(\frac{t_{1}-u_{1}}{t_{1}-z_{j}^{\prime}}\right)<\pi \quad \forall j=1,2, \ldots, n-1,
$$

and

$$
0<\arg \left[\left(\alpha_{j}\right) \cdot \prod_{t=1}^{n_{j}-1}\left(\frac{t_{1}-b_{j t}}{t_{1}-u_{1}}\right) \cdot \prod_{s=1}^{n_{j}}\left(\frac{t_{1}-u_{1}}{t_{1}-a_{j s}}\right)\right]<\pi, \quad 1<j<p .
$$


Consequently, the imaginary part of each term on the left-hand side of equation (2.16) is positive, which contradicts the fact that $t_{1}$ satisfies equation (2.16). Hence, all the zeros of $V(z)$ also lie on or inside the circle $C$.

This completes the proof of Theorem (2.3).

An immediate consequence of Theorem (2.3) is the following result, exclusively in terms of the differential equation (1.1).

COROLlary (2.4). Let $E$ be an ellipse with centre at the point c, semimajor axis as $a$, and eccentricity e. Let $0 \leqslant \gamma<\pi / 2$ and

$$
\lambda=\max \left\{1,\left(1-e^{2} / 2\right)^{1 / 2} \sec \gamma\right\} .
$$

If $\left|\arg \alpha_{j}\right| \leqslant \cos ^{-1}(1 / \lambda)$ for every $j=1,2, \ldots, p$ and if all the points $a_{j}$ $(1 \leqslant j \leqslant p)$ lie on or inside $E$, then the zeros of each Stieltjes polynomial $S(z)$ and the zeros of each Van Vleck polynomial $V(z)$, associated with the differential equation (1.1), lie in the circular region $|z-c| \leqslant \lambda a$.

Proof. In Theorem (2.3), if we take all $n_{j}$ 's to be 1 (i.e. $q=1$ ), then the differential equation (1.2) reduces to the differential equation (1.1), with the constants $a_{j}$ corresponding to the constants $a_{j 1}$, and Corollary (2.4) is then obvious.

The following corollary is a special case of Theorem (2.3) for circles.

COROLlaRy (2.5). If $\left|\arg \alpha_{j}\right| \leqslant \gamma<\pi / 2$ for every $j=1,2, \ldots, p$ and if all the points $a_{j s}, b_{j t}$ (occurring in the differential equation (1.2)) lie on or inside the circle $C:|z-c|=a$, then the zeros of each Stieltjes polynomial and the zeros of each Van Vleck polynomial, associated with the differential equation (1.2), lie in the circular region $|z-c| \leqslant a \sec [\{(q-1) \pi+\gamma\} /(2 q-1)]$.

Proof. In Theorem (2.3), if we take $e=0$ (i.e. if $E$ is taken as the circle $C$ ), then $\lambda=\sec \gamma$, and the corollary follows at once.

If we put $q=1$ in Corollary (2.5), we deduce the following well-known result due to Marden [1, Theorem (9.1)], [2], the proof being similar to that of Corollary (2.4).

Corollary (2.6). In the differential equation (1.1), if $\left|\arg \alpha_{j}\right| \leqslant \gamma<\pi / 2$ for every $j=1,2, \ldots, p$ and if all the points $a_{j}$ lie on or inside a circle $C$ : $|z-c|=a$, then the zeros of each Stieltjes polynomial and the zeros of each Van Vleck polynomial, associated with the differential equation (1.1), lie on or inside a concentric circle $C^{\prime}:|z-c|=a \sec \gamma$.

ReMARK. It may be noted that the theorem as stated by Marden [1, Theorem $(9,1)],[2]$ is, in fact, the same as Corollary (2.6) for the case when the centre $c=0$. But the same proof as given by him is valid also for the general case when the centre $c$ is not necessarily at the origin. 
As an application of Theorem (2.3), we prove

THEOREM (2.7). Let $0 \leqslant \gamma<\pi / 2$ and $\mu=\max \{1,(\sec \gamma) / \sqrt{2}\}$. If $\left|\arg \alpha_{j}\right|$ $\leqslant \cos ^{-1}(1 / \mu)$ for every $j=1,2, \ldots, p$ and if all the points $a_{j s}, b_{j t}$ (occurring in equation (1.2)) lie in the real interval $[-a, a]$, then the zeros of each Stieltjes polynomial $S(z)$ and the zeros of each Van Vleck polynomial $V(z)$, associated with the differential equation (1.2), lie in

$$
|z| \leqslant a \sec \left[\left\{(q-1) \pi+\cos ^{-1}(1 / \mu)\right\} /(2 q-1)\right],
$$

where $q=\max \left\{n_{1}, n_{2}, \ldots, n_{p}\right\}$.

Proof. Let $S_{e}$ denote the closed interior of an ellipse $E_{e}$ with centre at the origin, semimajor axis as $a$, and eccentricity $e$, and let

$$
\lambda_{e}=\max \left\{1,\left(1-e^{2} / 2\right)^{1 / 2} \sec \gamma\right\} \text {. }
$$

Then $[-a, a]=\bigcap_{e \in[0,1)} S_{e}$ and $\left(1-e^{2} / 2\right)^{1 / 2} \cdot \sec \gamma$ decreases continuously and monotonically to $(1 / \sqrt{2}) \cdot \sec \gamma$ as $e$ increases in $[0,1)$. Obviously, then $[-a, a] \subseteq S_{e}$ for every $e \in[0,1)$ and $\lambda_{e}$ decreases continuously and monotonically to $\mu$ as $e$ increases in $[0,1)$ (easy to verify this statement for the cases when $\gamma \leqslant \pi / 4$ and $\gamma>\pi / 4$ respectively). Therefore,

$$
\left|\arg \alpha_{j}\right| \leqslant \cos ^{-1}(1 / \mu) \leqslant \cos ^{-1}\left(1 / \lambda_{e}\right) \quad \forall e \in[0,1)
$$

and

(2.18) $a_{j s}, b_{j t} \in S_{e} \quad \forall e \in[0,1), \quad 1 \leqslant j \leqslant p ; 1 \leqslant s \leqslant n_{j} ; 1 \leqslant t \leqslant n_{j}-1$.

In view of (2.17) and (2.18), we can apply Theorem (2.3) and conclude that the zeros of each $S(z)$ and of each $V(z)$ lie in

$$
|z| \leqslant a \sec \left[\frac{(q-1) \pi+\cos ^{-1}\left(1 / \lambda_{e}\right)}{(2 q-1)}\right]=R_{e} \quad \text { (say) } \forall e \in[0,1) .
$$

Since $\lambda_{e}$ decreases continuously and monotonically to $\mu$ as $e$ increases in [0, 1), we conclude that $R_{e}$ decreases continuously and monotonically to

$$
a \sec \left[\left\{(q-1) \pi+\cos ^{-1}(1 / \mu)\right\} /(2 q-1)\right]=R \text { (say). }
$$

Finally, it is easy to see that the zeros of each $S(z)$ and those of each $V(z)$ lie in $|z| \leqslant R$. For, otherwise, it would contradict that the disc $|z| \leqslant R_{e}$ contains all the zeros of $S(z)$ and those of $V(z)$ for all values of $e$. This completes our proof.

The following corollary is an immediate consequence of the above theorem for the case when $q=1$ (use the same explanation as in the proof of Corollary (2.4)). 
Corollary (2.8). Let $0<\gamma<\pi / 2$ and $\mu=\max \{1,(1 / \sqrt{2}) \sec \gamma\}$. In equation (1.1), if $\left|\arg \alpha_{j}\right|<\cos ^{-1}(1 / \mu)$ for every $j=1,2, \ldots, p$ and if all the points $a_{j}$ lie in the real interval $[-a, a]$, then the zeros of each Stieltjes polynomial $S(z)$ and the zeros of each Van Vleck polynomial V(z), associated with the differential equation (1.1), lie in $|z| \leqslant \mu a$.

Finally, it may be remarked that the part of our Theorem (2.3) that concerns Stieltjes polynomials may be regarded as a generalization of Lucas' theorem $\left[1\right.$, Theorems $\left.(6,1),(6,1)^{\prime},(6,2)\right]$ to systems of partial fraction sums.

\section{REFERENCES}

1. M. Marden, Geometry of polynomials, 2nd ed., Math. Surveys, No. 3, Amer. Math. Soc., Providence, R. I., 1966. MR 37 \# 1562.

2. , On Stieltjes polynomials, Trans. Amer. Math. Soc. 33 (1931), 934-944.

3. E. Heine, Handbuch der Kugelfuntionen, Bd. I, 2nd ed., Springer, Berlin, 1878, pp. 472-476.

4. F. Lucas, Propriétés géométriques des fractions rationnelle, C. R. Acad. Sci. Paris 77 (1874), 431-433; ibid. 78 (1874), 140-144; ibid. 78 (1874), 180-183; ibid. 78 (1874), 271-274.

5. __ Géométrie des polynômes, J. Ecole Polytech. (1) 46 (1879), 1-33.

6. __ Statique des polynômes, Bull. Soc. Math. France 17 (1888), 2-69.

Department of Mathematics, Aligarh Muslim University, Aligarh 202001, U. P., India 